\title{
IMPACTO DEL MANEJO FORESTAL EN LA CONSERVACIÓN DE LA BIODIVERSIDAD EN LA REGIÓN AMAZONAS
}

\author{
IMPACT OF FOREST MANAGEMENT IN THE CONSERVATION OF BIODIVERSITY \\ IN THE AMAZON REGION
}

${ }^{1}$ Vitoly Becerra Montalvo a ${ }^{\text {y }}{ }^{1}$ Sonia Medina Díaz a

\section{RESUMEN}

Con el objetivo de establecer la relación entre el manejo forestal y la conservación de bosques en el nororiente peruano, se realizó un estudio en las provincias de Bagua y Condorcanqui en la región de Amazonas, considerándose la metodología de análisis de contenidos; en el cual, se consideró dos Títulos Habilitantes para personas naturales y dos para personas jurídicas, en este último caso se trata de Comunidades Nativas, ambos de la jurisdicción de la Región Amazonas. Se relacionó las directrices de Gestión Forestal Sostenible (UICN, 2010) tales como: Observar las leyes y planes nacionales y sus prácticas, establecer un plan de gestión forestal en el que se expliciten claramente los objetivos de conservación de la biodiversidad y su cumplimiento, plantaciones forestales, cumplimiento silvicultural, y asegurar que la gestión forestal conserve o acreciente la biodiversidad y garantice que se continúen cumpliendo funciones forestales, 01, 02, 10 y 11 respectivamente. Para la obtención de los Títulos Habilitantes todos los titulares cumplen con las directrices 01,02 y 10; sin embargo la directriz 11 que es la que valida la conservación de la biodiversidad no es exigida en los documentos de gestión para bosques de propiedad de personas naturales o privados, concluyendo que el manejo forestal realizado dentro de un marco normativo legal y técnico, que toman en cuenta los conocimientos y prácticas de manejo forestal de las comunidades nativas, garantizan la conservación de la biodiversidad, y por lo tanto la sostenibilidad del sector forestal.

Palabras clave: Manejo forestal sostenible, conservación de bosques, madera, productos forestales.

\section{ABSTRACT}

In order to establish the relationship between forest management and forest conservation in northeastern Peru, a study was conducted in the provinces of Bagua and Condorcanqui in the Amazon region, considering the content analysis methodology; in which two Authorization certificates for individuals and two, in for native communities are considered, both from the jurisdiction of the Amazon Region. Guidelines for Sustainable Forest Management (IUCN, 2010) were related: To I laws and national plans and practices, a forest management plan that clearly explicites objectives of biodiversity conservation and compliance, fore's Plantations, silvicultural compliance and ensure that forest management preserved and increased biodiversity and ensure that they continue to fulfill forest functions, 01, 02, 10 and 11 respectively. For obtaining the authorization certificates all holders meet with the guidelines 01, 02 and 10; however Guideline 11 that validates the conservation of biodiversity is not required in the documents management for forests natural or private owned by persons, concluding that forest management conducted within a legal and technical regulatory framework, taking into has knowledge and forest management practices of native communities, guarantee the conservation of biodiversity, and therefore the sustainability of the forest sector.

Keywords. Sustainable forest management, forest conservation, timber forest products.

Universidad Nacional de Jaén

Ingeniero Forestal 


\section{INTRODUCCIÓN}

El concepto de un sector forestal legal, regulado y manejado de manera sostenible ha sido entendido y adoptado por muchos gobiernos, ONGs y forestales como un equilibrio posible entre la conservación total (el enfoque de "no tocar") y el control de la explotación desmedida (el enfoque de "minería de madera"). Esta concepción intenta conciliar las inquietudes ambientales globales con los intereses y necesidades socioeconómicos de la sociedad, al tiempo que mantiene el equilibrio entre los intereses de los propietarios de tierras y las compañías forestales, con la necesidad de la conservación y la protección del medio ambiente (Greenpeace. 2011).

En la región nor oriental del Perú constituida por las provincias de Bagua y Condorcanqui se han venido implementando actividades de aprovechamiento forestal generalmente con fines maderables bajo diversos enfoques según la normatividad vigente en su contexto. Se analizó la correlación del manejo forestal con la conservación de los bosques establecidos en aquellos que cuentan con título habilitante, y que luego haya sido inspeccionado.

El manejo forestal sostenible es un concepto dinámico en evolución que tiene por objetivo mantener y aumentar el valor económico, social y medioambiental de todos los tipos de bosques, en beneficio de las generaciones presentes y futuras (ONU, 2008).

Es importante diferenciar entre enfoques de manejo que se limitan a la sostenibilidad del flujo de madera utilizable producida por una superficie dada de terreno (manejo forestal de rendimiento sostenido) y enfoques que procuran la sostenibilidad tanto del rendimiento como de la integridad ecológica básica del bosque (manejo forestal sostenible) (Darby, 1999).

Conceptualmente, la clave para el manejo forestal sostenible radica en no cortar más de lo que el bosque produce al crecer y garantizar que el aprovechamiento no altere la composición biológica ni la estructura física del bosque a largo plazo (si bien serán necesarias ciertas alteraciones de corto plazo para asegurar la regeneración de especies heliófitas) (Fredericksen, 1998).

Un buen aprovechamiento forestal debe basarse en la calidad y sensibilidad de la gestión forestal. No se puede anteponer los intereses de la explotación al riesgo de producir daños y perjuicios al bosque; se busca práctica y únicamente una mejora en la productividad económica, sin tener en cuenta las posibles disfunciones que se le puede ocasionar al mismo (López, 2013).

El manejo forestal sostenible requiere, a la vez, delimitar la corta de árboles e invertir en reemplazarlos, al promover la regeneración natural de las especies cosechadas o sembrando plántulas de las mismas (ITTO, 1990).

Organizaciones como Greenpeace (Greenpeace 2011) y World Wildlife Fund (WWF, 2010), conocidas por su compromiso con la protección de la naturaleza y la vida salvaje, reconocen, hoy por hoy, que la madera legal proveniente de bosques manejados de forma sostenible es una poderosa herramienta de conservación y están dedicando esfuerzos crecientes a la creación y apoyo de canales de madera legal de bosques naturales.

La relación entre aprovechamiento y conservación de los bosques es uno de los problemas más graves. No obstante, esto no significa que haya conciencia social ni planes específicos adecuados para una ordenación forestal sostenible. Todo ello hace más necesaria y urgente la intervención y concienciación de los agentes estatales, institucionales y locales (Urquijo, 1999).

El objetivo es establecer la relación que existe entre el manejo forestal y la conservación de bosques en el nororiente peruano. 


\section{MATERIALY MÉTODOS}

El estudio se realizó en las provincias de Bagua y Condorcanqui en la región de Amazonas .

Se consideró la metodología de análisis de contenidos; con dos Títulos Habilitantes para personas naturales y dos para personas jurídicas, en este último caso se trata de Comunidades Nativas, ambos de la jurisdicción de la Región Amazonas.

El resumen de los casos analizados se muestra en el cuadro 1 de las directrices de Gestión Forestal Sostenible en el cuadro 2.

Cuadro 1. Relación de Títulos Habilitantes e Informes de Supervisión Final de Ejecución del Manejo Forestal

\begin{tabular}{|c|c|c|c|}
\hline CÓDIGO & $\begin{array}{l}\text { TITULO HABILITANTE } \\
\text { APROBADO CON }\end{array}$ & $\begin{array}{c}\text { INSPECCIÓN } \\
\text { FINAL APROBADO } \\
\text { CON }\end{array}$ & TITULAR \\
\hline 01 & $\begin{array}{l}\text { RESOLUCIÓN } \\
\text { DIRECTORAL REGIONAL } \\
\text { № 229-2013- } \\
\text { GRA/GRDE/DRAD/D } \\
\end{array}$ & $\begin{array}{l}\text { INFORME N }{ }^{\circ} 268- \\
\text { 2014-OSINFOR/06.2.1 }\end{array}$ & $\begin{array}{l}\text { COMUNIDAD } \\
\text { NATIVA }\end{array}$ \\
\hline 02 & $\begin{array}{l}\text { RESOLUCIÓN } \\
\text { DIRECTORAL REGIONAL } \\
\text { No 099-2011- } \\
\text { GRA/GRDE/DRAD/D } \\
\end{array}$ & $\begin{array}{l}\text { INFORME No } 193- \\
\text { 2013-OSINFOR/06.2.1 }\end{array}$ & $\begin{array}{l}\text { COMUNIDAD } \\
\text { NATIVA }\end{array}$ \\
\hline 03 & $\begin{array}{l}\text { RESOLUCIÓN } \\
\text { DIRECTORAL REGIONAL } \\
\text { No }^{\circ} 202-2013- \\
\text { GRA/GRDE/DRAD/D } \\
\end{array}$ & $\begin{array}{l}\text { INFORME N }{ }^{\circ} 349- \\
\text { 2013-OSINFOR/06.2.1 }\end{array}$ & $\begin{array}{l}\text { PERSONA } \\
\text { NATURAL }\end{array}$ \\
\hline 04 & $\begin{array}{l}\text { RESOLUCIÓN } \\
\text { DIRECTORAL REGIONAL } \\
\text { No } 254-2012-^{\text {GRA/GRDE/DRAD/D }} \\
\end{array}$ & $\begin{array}{l}\text { INFORME N }{ }^{\circ} 348- \\
\text { 2013-OSINFOR/06.2.1 }\end{array}$ & $\begin{array}{l}\text { PERSONA } \\
\text { NATURAL }\end{array}$ \\
\hline
\end{tabular}

Fuente: ARA-GORE AMAZONAS, 2015

Cuadro 2. Directrices de Gestión Forestal Sostenible

\begin{tabular}{ccl}
\hline $\mathbf{N}^{\circ}$ & CÓDIGO & \multicolumn{1}{c}{ DIRECTRIZ } \\
\hline 1 & 01 & Observar las leyes y planes nacionales y sus prácticas \\
\hline 2 & 02 & $\begin{array}{l}\text { Establecer un plan de gestión forestal en el que se } \\
\text { expliciten claramente los objetivos de conservación } \\
\text { de la biodiversidad y su cumplimiento }\end{array}$ \\
\hline 3 & 10 & Plantaciones forestales, cumplimiento silvicultural \\
\hline 4 & 11 & $\begin{array}{l}\text { Asegurar que la gestión forestal conserve o acreciente } \\
\text { la biodiversidad y garantice que se continúen } \\
\text { cumpliendo funciones forestales }\end{array}$ \\
\hline
\end{tabular}

Fuente: UICN, 2010

\section{RESULTADOS Y DISCUSIONES}

\section{Resultados}

Luego de la revisión tanto de los documentos de Gestión del Título Habilitante (Plan de Manejo Forestal para el caso de Comunidades Nativas y Plan Operativo Anual para el caso de personas naturales), así como los informes de supervisión final, se tienen los resultados resumidos en la siguiente tabla:
Cuadro 03. Cumplimiento de las Directrices de Gestión Forestal Sostenible en el Documento de Gestión.

\begin{tabular}{cccccc}
\hline \multirow{2}{*}{$N^{\circ}$} & \multirow{2}{*}{$\begin{array}{c}\text { TÍTULO } \\
\text { HABILITANTE }\end{array}$} & \multicolumn{3}{c}{ CUMPLIMIENTO DE LA } \\
\cline { 3 - 6 } & DIRECTRIZ/CÓDIGO \\
\hline 1 & 01 & SI & 02 & $\mathbf{1 0}$ & $\mathbf{1 1}$ \\
\hline 2 & 02 & SI & SI & SI & SI \\
\hline 3 & 03 & SI & SI & SI & SI \\
\hline 4 & 04 & SI & SI & SI & NO \\
\hline
\end{tabular}

En los documentos de gestión se encuentran contemplados las directrices de código 01,02 y 10 en un $100 \%$; sin embargo el código 11 solo se encuentra contemplado en un $75 \%$ de los Títulos Habilitantes.

Cuadro 04. Cumplimiento de las Directrices de Gestión Forestal Sostenible en el Informe de supervisión final

\begin{tabular}{cccccc}
\hline \multirow{2}{*}{$\mathbf{N}^{\circ}$} & \multirow{2}{*}{$\begin{array}{c}\text { TÍTULO } \\
\text { HABILITANTE }\end{array}$} & \multicolumn{3}{c}{$\begin{array}{c}\text { CUMPLIMIENTO DE LA } \\
\text { DIRECTRIZ/CÓDIGO }\end{array}$} \\
\cline { 2 - 6 } & $\mathbf{0 1}$ & $\mathbf{0 2}$ & $\mathbf{1 0}$ & $\mathbf{1 1}$ \\
\hline 1 & 01 & NO & NO & NO & SI \\
\hline 2 & 02 & NO & NO & SI & SI \\
\hline 3 & 03 & SI & SI & SI & SI \\
\hline 4 & 04 & SI & SI & SI & SI \\
\hline
\end{tabular}

Las directrices 01 y 02 no se cumplen en el 100\% de los Títulos Habilitantes pertenecientes a comunidades nativas, las mismas que solo cumplen totalmente la directriz 11 y en un $50 \%$ la directriz 10. Los Títulos Habilitantes pertenecientes a personas naturales cumplen al $100 \%$ las directrices evaluadas, según lo determinan los informes de supervisión realizado por OSINFOR.

\section{Discusiones}

Para la obtención de los Títulos Habilitantes, los titulares solicitantes elaboran un documento de gestión según sea el caso Plan de Manejo forestal para el caso de comunidades nativas, o Plan Operativo Anual para el caso de personas naturales que van a realizar manejo forestal en bosques de su propiedad. Estos documentos tienen Términos de Referencia aprobados por la Autoridad Forestal Nacional que para el caso actualmente es el SERFOR. Según estos términos de referencia puede verse que en el caso 
de comunidades nativas, se contempla un cumplimiento de todas las directrices analizadas, buscando garantizar la conservación de la biodiversidad; sin embargo, para el caso de personas naturales no considera la directriz 11 , esto debido a que se trata de un predio de propiedad privada, donde el titular puede implementar alguna actividad productiva diferente a la forestal, tomando en cuenta desde luego la aptitud de uso mayor de su suelo y cumpliendo todas las normas para el cambio de uso del suelo; sin embargo la Evaluación de los Ecosistemas del Milenio afirman que avanzan las zonas dedicadas a la agricultura y el pastoreo, muchas veces a costa de los bosques, lo cual revela que en cerca del $70 \%$ de los países estudiados las tierras agrícolas se están expandiendo, el avance de la frontera agrícola ha tenido un impacto particularmente fuerte en las regiones de bosques tropicales, donde se prevé que las tierras de pastoreo y cultivo continúen creciendo en los próximos 30 a 50 años (EEM 2005); además la ONU (2008) afirma que alrededor del $40 \%$ de la economía mundial depende directamente de la biodiversidad, en particular los sectores agrícola y forestal.

Si bien no es obligatorio en los términos de referencia de los documentos de gestión actividades de conservación de la biodiversidad, en los casos analizados vemos que si se han considerado acciones tendientes a lograr esto, sobre todo en el aspecto silvicultural; sin embargo al ser el documento de gestión de un muy corto horizonte (un año), la conservación de la biodiversidad no está garantizada, confirmando lo señalado por Urquijo (1999) quien indica que la relación entre aprovechamiento y conservación de los bosques es uno de los problemas más importantes a tomar en cuenta.

Por el contrario en las comunidades nativas, el documento de gestión contempla actividades que garantizan la conservación de labiodiversidad y su aplicación es en un horizonte de mediano a largo plazo, ya que el Banco Mundial (2004) calcula que aproximadamente 60 millones de personas pertenecientes a pueblos indígenas dependen casi totalmente de los bosques, unas 350 millones de personas derivan gran parte de su subsistencia e ingresos de los bosques y cerca de 1.200 millones viven de sistemas de cultivo agrosilvícolas.

Del análisis de los resultados obtenidos en las supervisiones realizadas por OSINFOR, según sus informes correspondientes, estos son diferentes para los casos de comunidades nativas y titulares de personas naturales pese a que la WWF (2009) señala que las comunidades indígenas, manejan sosteniblemente áreas ubicadas en el ámbito del Plan General del Manejo Forestal (PGMF) de acuerdo a las normas legales vigentes $\mathrm{y}$ del ordenamiento territorial de las comunidades.

No hay una concordancia entre la parte legal normativa e incluso metodológica de lo que es el manejo forestal, y el concepto que las comunidades nativas tienen al respecto. Los términos de referencia para los documentos de gestión no contemplan metodologías de manejo forestal que desarrollan las comunidades nativas en su relación ancestral con el bosque, estos están parametrados a ensayos y metodologías desarrolladas por la silvicultura tropical reciente e incipiente del colono o mestizo que trata de manejar los bosques de manera sostenible.

Esta diferencia of falta de concordancia hace que las comunidades nativas no sean fieles cumplidores de la normativa legal del manejo forestal, y aun si aceptan los documentos de gestión forestal, les es muy difícil cumplirlo, ya que no se ajusta a sus conocimientos y prácticas ancestrales de manejo. Los resultados se reflejan en los informes de supervisión, donde el 100\% de las comunidades nativas no han cumplido con la implementación del documento de gestión, trasgrediendo la legislación forestal (directriz 01 y 02 ). 
Por el contrario los Títulos Habilitantes cuyos titulares son personas naturales dan como resultado un cumplimento de la normativa legal y técnica, por estar estos titulares mejor relacionados con los criterios y metodologías que contemplan los términos de referencia para elaborar los documentos de gestión; sin embargo AIDER (2005) menciona que es difícil estimar la proveniencia de la madera, por la poca transparencia de los datos y la variabilidad de formas de ilegalidad, ya que se estima que entre el 50 y el 80 por ciento de la madera extraída es de origen ilegal y estos actores compiten en el mercado con precios muy bajos.

Sin embargo a pesar que las comunidades nativas no cumplen con la implementación completa de los documentos de gestión, la supervisión indica que el bosque sigue cumpliendo sus funciones ecológicas, incluso en el caso que no se cumpla la aplicación del sistema silvicultural aprobado. Además tomando en cuenta que el documento de gestión tiene un horizonte de mediano o largo plazo, es de esperar que la conservación de la biodiversidad este mas garantizada en estas comunidades nativas a diferencia de los casos de los titulares individuales o privados.

\section{CONCLUSIONES}

Para la obtención de los Títulos Habilitantes todos los titulares cumplen con las directrices 01 , 02 y 10 ; sin embargo la directriz 11 que es la que valida la conservación de la biodiversidad no es exigida en los documentos de gestión para bosques de propiedad privada o de personas naturales.

El manejo forestal por parte de los titulares considerados como personas naturales o privada han cumplido la implementación de todas las directrices analizadas; sin embargo debido al corto periodo de implementación del documento de gestión, esto no garantiza la conservación de la biodiversidad.

El manejo forestal realizado por las comunidades nativas a pesar de no cumplir fielmente las directrices 01 y 02 garantiza en mayor medida la conservación de la biodiversidad en un largo plazo.

La normativa forestal y los documentos de gestión utilizados para el manejo forestal no se ajustan a la forma como las comunidades nativas vienen gestionando sus bosques desde tiempos ancestrales, y esto genera el incumplimiento obligado de las directrices 01 y 02 analizadas.

Como conclusión final tenemos que el manejo forestal realizado dentro de un marco normativo legal y técnico, con los conocimientos y prácticas de manejo forestal de las comunidades nativas, garantizan la conservación de la biodiversidad, y por lo tanto la sostenibilidad del sector forestal.

\section{AGRADECIMIENTOS}

A la Autoridad Regional Ambiental de la Región Amazonas por brindar la Información Oficial para realizar los análisis de contenido en la ejecución de la presente investigación.

A la Escuela de Post Grado por la orientación en la ejecución de la presente investigación a través de la Doctora Zoila Cruz Burga.

\section{REFERENCIAS BIBLIOGRÁFICAS}

AIDER, 2005. Asociación para la Investigación y el Desarrollo Integral. El proceso del manejo forestal responsable en la comunidad nativa de Callería. Análisis de la contribución económica y social. WWF, Perú - USAID. Ed. Argentina.76P

ARA, 2015. Autoridad Regional Ambiental del Gobierno Regional de Amazonas. Resolución Directoral Regional $\mathrm{N}^{\circ}$ 229-2013GRA/GRDE/DRAD/D. Informe $\mathrm{N}^{\circ}$ 268-2014OSINFOR/06.2.1

ARA, 2015. Autoridad Regional Ambiental del Gobierno Regional de Amazonas. Resolución Directoral Regional $N^{\circ}$ 099-2011GRA/GRDE/DRAD/D. Informe $N^{\circ}$ 193-2013OSINFOR/06.2.1 
ARA, 2015. Autoridad Regional Ambiental del Gobierno Regional de Amazonas. Resolución Directoral Regional No 202-2013GRA/GRDE/DRAD/D. Informe $N^{\circ}$ 349-2013OSINFOR/06.2.1

ARA, 2015. Autoridad Regional Ambiental del Gobierno Regional de Amazonas. Resolución Directoral Regional No 254-2012-GRA/GRDE /DRAD/D. Informe $N^{\circ} 348-2013-O S I N F O R /$ 06.2 .1

Banco Mundial. 2004. Sustaining Forests: A Development Strategy. Washington, D.C.: Banco Mundial. 68P

Darby, 1999. La Certificación y el Manejo Forestal Sostenible en Bolivia. Documento Técnico 79/1999. Objetivo Estratégico de Medio Ambiente-USAID-Bolivia.

EEM, 2005. Evaluación de los Ecosistemas del Milenio. Ecosystems and Human Well-Being: Policy Responses. Volumen 3. Cap. 8. Island Press, Washington, DC. 155P

Fredericksen, TS. 1998. Limitations of lowintensity selective logging for sustainable tropical forestry. BOLFOR Technical Document 68/1998.

Greenpeace. 2011. Working with forest communities in Greenpeace.

ITTO, 1990. Guidelines for sustainable management of natural tropical forests. International Tropical Timber Organization, Yokohama, Japan.

López, 2013. Conservación de bosques. Agencia de Medio Ambiente de Madrid. C/Princesa, 3-9 ${ }^{\circ}$. 28008 -Madrid-España.

ONU, 2008. Organización de las Naciones Unidas. Resolución aprobada por la Asamblea General 62/98: Instrumentos jurídicamente no vinculantes sobre todos los tipos de bosques. Disponible en: http://daccess-ddsny.un.org/doc/UNDOC/GEN/N07/469/68/PDF/ N0746968.pdf?OpenElement
UICN, 2010. Unión Internacional para la Conservación de la Naturaleza. Guía de Buenas Prácticas. Gestión Forestal Sostenible, Biodiversidad y Medios de Vida.

Urquijo, 1999. MAGA. Ministerio de Agricultura, Ganadería y Alimentación. ETSI Agrónomos. UPM. Guatemala. 65-68P

WWF. 2009. Fortalecimiento del manejo forestal sostenible en territorios amazónicos de pueblos indígenas en el Perú. Ejecutado por Cesvi y financiado por UNIÓN EUROPEA - Cesvi WWF.

WWF. 2010. GFTN (Global Forest \& Trade Network) annual report 2010. World Wildlife Fund. http://assets.panda.org

\section{CORRESPONDENCIA}

Vitoly Becerra Montalvo

Avenida Mesones Muro No 889 - Jaén, Cajamarca, Perú

vitoly@gmail.com 